\title{
Overexpression of S-Adenosylmethionine Synthetase Gene from Pyropia tenera Enhances Tolerance to Abiotic Stress
}

\author{
Hyun-Ju Hwang, Jin-Woo Han, Hyun Dae Hong, Jong Won Han* \\ Department of Genetic Resources Research, National Marine Biodiversity Institute of Korea, Seocheon 33662, Korea
}

\begin{abstract}
Pyropia tenera is an intertidal red alga of commercial significance owing to its popularity as a health-promoting seafood product. This alga grows in marine environments and is frequently exposed to high salinity and osmotic stress, which impact its growth. Therefore, the enhancement of stress tolerance in $P$. tenera is critical. In the present work, we aimed to elucidate the mechanisms underlying abiotic stress tolerance in this species; specifically, we identified the $P$. tenera S-adenosylmethionine synthetase-encoding gene (PtSAMS) and characterized its biological function. This gene, which is known to play a role in stress tolerance in other plants, was cloned and overexpressed in Escherichia coli under high-salinity conditions. The PtSAMS gene was found to encode a 385-amino-acid protein with a molecular weight of $41.8 \mathrm{kDa}$. In silico sequence alignment and phylogenetic analysis of the PtSAMS amino acid sequence showed that the encoded protein comprises three conserved domains and two motifs that are highly conserved in other plants. Growth assay results indicated that PtSAMS-overexpressing E. coli cells exhibit enhanced tolerance to salt stress. The results suggest that PtSAMS expression is induced by a combination of ion toxicity and osmotic stress resulting from exposure to high salinity in marine environments, and that this gene is expressed at housekeeping levels owing to growth in such conditions. The findings suggest that PtSAMS could be used as a potentially valuable bioresource with utility in the genetic engineering of salt stress-tolerant crop plants.
\end{abstract}

Keywords S-adenosylmethionine synthetase (SAMS), Pyropia tenera, Abiotic stress, Salinity, Recombinant protein, Tolerance

\section{INTRODUCTION}

Algae have been consumed as edible seaweed in Asia for thousands of years. Pyropia (Rhodophyta) have been cultivated since the $17^{\text {th }}$ century in Korea, Japan, and China and represent commercially valuable seafood. Pyropia, which is highly popular owing to its delicious taste, contains high levels of protein and is rich in essential amino acids and vitamins (Blouin et al. 2011). The market for Pyropia is worth about 7 trillion, and its exports have experienced recent growth. It expected that the Pyropia market size would continue to increase as a result of its recognition as a health-beneficial food product.

The environment in which seaweed is cultivated is exposed to harsh marine conditions. Aquaculture produc- tivity is affected by temperature, rainfall, laver disease, and other factors. Therefore, the identification of rapidly growing or disease-resistant seaweed species is critical.

Salinity is one of the major abiotic factors that limit crop growth and productivity. Salt stress results in excessive ion toxicity, oxidative stress, and physiological drought, consequently causing disruption of cell organelles and their metabolism, eliciting nutrient imbalance in the plant, and reducing its osmotic potential (Tester and Bacic 2005). These effects lead to arrest of growth and development of the plant, thereby limits its survival.

S-Adenosylmethionine synthetase (SAMS) catalyzes the conversion of adenosine 5'-triphosphate (ATP) and methionine into S-adenosylmethionine (SAM) (Pajares and Markham 2011). SAM plays a central role in diverse

Received November 1, 2017; Revised November 9, 2017; Accepted November 10, 2017; Published December 1, 2017

*Corresponding author Jong Won Han, jwhan@mabik.re.kr, Tel: +82-41-950-0760, Fax: +82-41-950-0765 
biological processes, such as by serving as a methyl donor during transmethylation in plants, and acting as a common precursor in the biosynthesis of polyamines and ethylene (Kumar et al. 1997; Roeder et al. 2009). The SAMSencoding gene has been cloned from various species such as bacteria, yeasts, humans, animals, and plants. In plants, SAMS has been reported to play a role in developmental process and the stress response (Pulla et al. 2009). In Arabidopsis thaliana, the SAMS gene plays a role in seed germination, as shown in a previous study of MTO3 mutants that showed delayed germination and high level concentration of methionine phenotype Knockdown of the SAMS gene resulted in delayed flowering time and dwarf phenotype in rice (Boerjan et al. 1994; Li et al. 2011). Expression of SAMS is induced under low temperature in Arabidopsis, rice, and maize (Cui et al. 2005; Amme et al. 2006; Yang et al. 2006; Uvackova et al. 2012). Pisum sativum SAMS1 is expressed during pea development (Gómez-Gómez and Carrasco 1998). Overexpression of the stress-inducible gene GSSAMS2 enhances salt tolerance in transgenic Medicago sativa (Hua et al. 2012). Suaeda salsa inhabits saline or alkaline soil such as coastal salt-flats. Recently, overexpression of $S$. salsa SAMS was shown to increase salt tolerance in transgenic tobacco (Qi et al. 2010). Although Porphyra yezoensis SAMS (PySAMS) has been cloned (Yi et al. 2009), its function is still unknown.

Here, we reported the cloning and characterization of the SAMS (PtSAMS) gene in Pyropia tenera. In order to elucidate the function of PtSAMS, we additionally examined the expression of PtSAMS in E. coli under stress conditions. Functional analysis in E. coli suggested that PtSAMS plays a role in the tolerance to stress.

\section{MATERIALS AND METHODS}

\section{Plant materials}

The $P$. tenera strain used in this study was received from the Seaweed Research Center, National Research \& Development Institute. P. tenera was cultured at $10^{\circ} \mathrm{C}$ in a growth chamber with bubbling under $50 \mu \mathrm{mol} \cdot \mathrm{m}^{-2} \mathrm{~s}^{-1}$ and a photoperiod of 12 hour light and 12 hour dark. The culture media used was Provasoli's enrichment solution (PES); the media were changed every week (Kakinuma et al. 2016).

\section{RNA extraction, reverse transcription, and isolation of PtSAMS gene}

The PtSAMS sequence was obtained by performing a search of the NCBI (National Center of Biotechnology Information) database, based on the sequence of Porphyra yezoensis S-adenosylmethionine synthetase (SAMS) gene. Total RNA was extracted from P. tenera with Hybrid-R (GeneAll, Seoul, Korea) according to the manufacturer's guide. The first-strand cDNA was synthesized using amfiRivert cDNA Synthesis Platinum Master Mix (GenDEPOT, Texas, US). The full-length PtSAMS gene was isolated through RT-PCR using the PtSAMS-F (5'CACCATGGCAGCCATGAAG-3') and PtSAMS-R (5'ACGACGCTCTAGAGCTCAC-3') primers based on the P. yezoenesis sequence (GenBank accession: FJ404748) (Table 1).

\section{Cloning of PtSAMS gene into the pET28(b) expression vector and recombinant protein expression}

PtSAMS was amplified by PCR using the PtSAMSNdeI-F (5'-CCCCATATGGCAGCCATGAAGA-3') and PtSAMS-XhoI-R (5'-TCACTCGAGGAGCTCAAGC-3') primers with flanking restriction sites of NdeI and XhoI, respectively (Table 1). The PCR product was digested with NdeI and $\mathrm{XhoI}$ restriction endonucleases and cloned into pET28(b). The pET28(b)-PtSAMS plasmid was transformed into the E. coli strain BL21(DE3). The pET28(b) empty vector was used as a control in spot assay and liquid growth assay of stress treatment (Yadav et al. 2012).

\section{Sequence analysis}

PtSAMS and protein sequences of other SAMS of different species were searched in the NCBI database. Amino acid alignments were conducted using the ClustalW algorithm implemented in BioEdit (Thompson et al. 1994). The phylogenetic tree of the SAMS protein was constructed using the Geneious software 9.0.4. using a neighbor-joining (NJ) method with bootstrap set at 10,000 replicates. 
Table 1. Gene specific primers used in the study.

\begin{tabular}{|c|c|c|}
\hline Name & Sequence $\left(5^{\prime}-3^{\prime}\right)$ & Purpose \\
\hline PtSAMS-F & CACCATGGCAGCCATGAAG & For full length of PtSAMS gene \\
\hline PtSAMS-R & ACGACGCTCTAGAGCTCAC & For full length of $P t S A M S$ gene \\
\hline PtSAMS-NdeI-F & CCCCATATGGCAGCCATGAAGA $^{z)}$ & For protein expression \\
\hline PtSAMS-XhoI-R & TCACTCGAGGAGCTCAAGC & For protein expression \\
\hline PtSAMS-303-F & CCAGTCCCCTGAGATTGCTG & Probe for southern blot analysis \\
\hline PtSAMS-955-R & CTGAAATCGGCTCGGCAATG & Probe for southern blot analysis \\
\hline PtSAMS-406-F & GAGGACCTGATGCCCTTGAC & RT-qPCR \\
\hline PtSAMS-591-R & CTGCGTGCTGATGACAATCG & RT-qPCR \\
\hline$P t G A P D H-2771-\mathrm{F}$ & CGCCGAGTACATTGTCGAGT & RT-qPCR \\
\hline$P t G A P D H-3002-\mathrm{R}$ & GTACTTCTCGTGCAGCACCT & RT-qPCR \\
\hline
\end{tabular}

${ }^{z)}$ Bold letters indicate restriction enzyme sites.

\section{Southern blot analysis}

Genomic DNA was isolated from P. tenera using the DNeasy Plant Maxi-prep Kit (Qiagen, Hilden, Germany). Genomic DNA ( $5 \mu \mathrm{g})$ was digested with BamHI and KpnI, separated on $0.7 \%$ agarose gel, and transferred to Hybond-N + membrane. The membrane was treated by UV crosslinking to fix the genomic DNA. Probe DNA was prepared by PCR for a specific region of PtSAMS. The gene-specific primers used were PtSAMS-303-F (5'CCAGTCCCCTGAGATTGCTG-3') and PtSAMS-955-R (5'-CTGAAATCGGCTCGGCAATG-3') (Table 1). Probe labeling with dioxigenin (DIG), hybridization, and detection were carried out using the DIG High Prime DNA Labeling and Detection Starter Kit I (Roche, Basel, Switzerland) according to the manufacturer's instructions.

\section{Quantitative RT-PCR analysis}

cDNA from $P$. tenera was used as a template for qRT-PCR using PtSAMS gene-specific primers PtSAMS303-F and PtSAMS-955-R. Real time qPCR was performed using the Takara PCR system with a SYBER GREEN KIT (Takara, Shiga, Japan). PCR conditions were as follows: $94^{\circ} \mathrm{C}$ for 2 minutes, 40 cycles at $94^{\circ} \mathrm{C}$ for 30 seconds, $62^{\circ} \mathrm{C}$ for 30 seconds, $72^{\circ} \mathrm{C}$ for 30 seconds, and a final extension at $72^{\circ} \mathrm{C}$ for 10 minutes. The experiments were repeated three times independently. The PtGAPDH gene was used as an internal control under the same conditions. Primer sequences were as follows: PyGAPDH-2771-F (5'-CGCC GAGTACATTGTCGAGT-3') and $P y G A P D H-3002-\mathrm{R}$
(5'-GTACTTCTCGTGCAGCACCT-3') (Lee et al. 2015) (Table 1).

\section{Spot culture assay for analysis of the effects of stress} treatment

pET28(b)-PtSAMS/BL21(DE3) cells were grown in LB medium to $\mathrm{OD}_{600}=0.6$, and $0.1 \mathrm{mM}$ IPTG was added (Yadav et al. 2012). The inducted cells were grown for 5 hours at $37^{\circ} \mathrm{C}$. The cultured cells were dilouted to an $\mathrm{OD}_{600}$ of 0.6 , and then to $10^{-3}, 10^{-4}$, and $10^{-5}$. Ten micro liters from each dilution was spotted on to an LB-only plate, or an LB plate supplemented with $400 \mathrm{mM} \mathrm{NaCl}$ and $400 \mathrm{mM} \mathrm{KCl}$. The experiments were repeated three times independently.

\section{Liquid culture assay under stress treatment}

Growth rate analysis was tested in LB liquid medium or in $\mathrm{LB}$ medium supplemented with $\mathrm{NaCl}$ and $\mathrm{KCl}$. Then, $400 \mu \mathrm{L}$ of pET28(b)-PtSMAS recombinant or vector alone in E. coli BL21 cells were inoculated into $10 \mathrm{~mL}$ of LB liquid medium for overnight culture. The next day, the cultured cells were diluted to an $\mathrm{OD}_{600}$ of 0.6 and $0.1 \mathrm{mM}$ IPTG was added for induction. Cells were grown for 5 hours at $37^{\circ} \mathrm{C}$ and then were diluted to $\mathrm{OD}_{600}$ of 0.6 . Next, $500 \mu \mathrm{L}$ of cells were inoculated into $50 \mathrm{~mL}$ of $\mathrm{LB}$ medium containing $400 \mathrm{mM} \mathrm{NaCl}$, and $400 \mathrm{mM} \mathrm{KCl}$, or $\mathrm{LB}$ medium only, and cultured at $37^{\circ} \mathrm{C}$. Cells were harvested every 1 hour, until 12 hours, and $\mathrm{OD}_{600}$ values were measured. 


\section{RESULTS}

\section{Isolation and characterization of PtSAMS}

To isolate the PtSAMS, we designed primer sets based on the $P$. yezoensis sequence (Accession number: FJ404748) in the NCBI GenBank sequence database (Table 1). The PtSAMS gene was cloned from the cDNA library by PCR and sequenced (data not shown). Sequence analysis showed that the complete open reading frame of PtSAMS is $1,155 \mathrm{bp}$ in length, and is composed of one exon. The predicted PtSAMS protein comprises 385 amino acid residues with a calculated molecular weight of $41.8 \mathrm{kDa}$ and isoelectric point (PI) of 5.60.

\section{Sequence analysis and phylogenetic analysis of PtSAMS}

To further characterize the PtSAMS protein, we performed a conserved domain (CD) analysis through the GenomeNet Database Resources (http://www.genome.jp/ tools/motif/). The predicted PtSAMS protein consisted of three S-adenosylmethionine synthetase $\mathrm{N}$-terminal, central, and C-terminal domains. Furthermore, the predicted PtSAMS sequence contained two substrate-binding sites, as well as a site for ATP binding in the central domain and for Met binding in the C-terminal domain (Fig. 1a). The BioEdit software was used for comparison of the amino acid sequence of PtSAMS with SAMS from other representative Rhodophyta, Phaeophyta, and Chlorophyta (Fig. 1a). The results showed that PtSAMS shared higher homology with SAMS from plant species such as Galdieria sulphuraria (76\%), Ectocarpus siliculosus (70\%), Chlamydomonas reinhardtii (69\%), and A. thaliana (66\%). To investigate the evolutionary relationship of PtSAMS protein among SAMS proteins of other species, a phylogenetic tree was constructed using Geneious 9.0.4 software on the basis of the multiple amino acid sequences (Fig. 1b). GenBank accession numbers of the protein sequences are shown in Table 2. Phylogenetic tree analysis revealed that the PtSAMS of Bangiophyceae was grouped with red algae, showing the highest degree of clustering with Rhodophyta, Galdieria sulphuraria, and then with SAMS of diatoms and Phaeophyta, followed by Chlorophyta. Table 3 shows pairwise comparison of the amino acid sequences between PtSMAS and other species. The SAMS sequence was found to be highly conserved among all the different species.

a
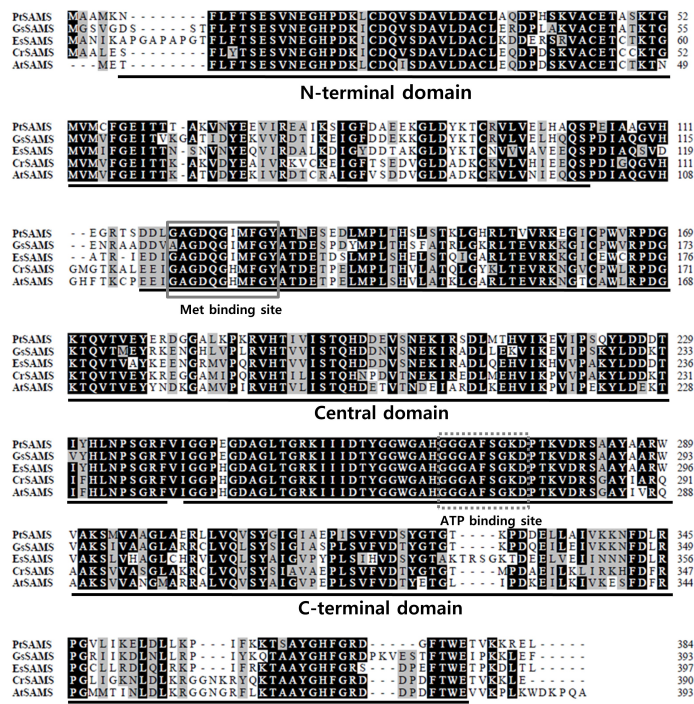

b

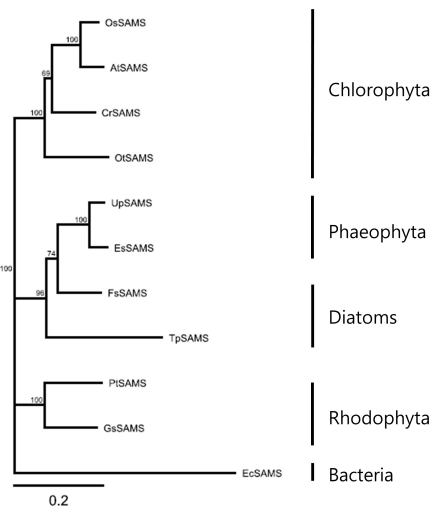

Fig. 1. Amino acid sequence alignment of PtSAMS homologs and phylogenetic tree of SAMS from various species. (a) Comparison of amino acid sequences of Pyropia tenera SAMS with the SAMS of other species: Galdieria sulphuraria (XP_005703068.1), Ectocarpus siliculosus (CBJ30117.1), Chlamydomonas reinhardtii (XP_001696661.1), and Arabidopsis thaliana (NP_171751.1); identical residues are shaded black, and similar residues are shaded gray. The domains are indicated by underlining. Met-binding motifs are indicated by boxes. Dotted boxes indicate ATP-binding sites. (b) Phylogenetic tree analysis using Geneious software using Jukes Cantor, Neighbor Joining tree building method with a 10,000 bootstrap repeat value. 
Table 2. GenBank and NCBI reference sequence accession numbers of SAMS sequences.

\begin{tabular}{llll}
\hline \hline \multirow{2}{*}{ Species } & Amino acid sequence length & NCBI reference sequence \\
\hline Rhodophyta & Pyropia yezoensis & 384 & ACJ98094.1 \\
& Galdieria sulphuraria & 393 & XP_005703068.1 \\
Phaeophyta & Ectocarpus siliculosus & 397 & CBJ30117.1 \\
& Undaria pinnatifida & 397 & AEK80411.1 \\
Chlorophyta & Ostreococcus tauri & 386 & XP_003083240.1 \\
& Chlamydomonas reinhardtii & 390 & XP_001696661.1 \\
& Arabidopsis thaliana & 393 & NP_171751.1 \\
Diatoms & Oryza sativa & 396 & XP_0022888854.1 \\
& Thalassiosira pseudonana & 450 & GAX17078.1 \\
Bacterial & Fistulifera solaris & 384 & AAA24164.1 \\
\hline
\end{tabular}

Table 3. Amino acid sequence identity matrix comparison of PtSMAS with SAMS of other species.

\begin{tabular}{lcrrrr}
\hline \hline & PtSAMS & GsSAMS & CrSAMS & EsSAMS & AtSAMS \\
\hline PtSAMS & $100 \%$ & $76.84 \%$ & $70 \%$ & $69.6 \%$ & $66.75 \%$ \\
GsSAMS & & $100 \%$ & $69.77 \%$ & $68.91 \%$ & $67.59 \%$ \\
CrSAMS & & & $100 \%$ & $65.92 \%$ & $81.14 \%$ \\
EsSAMS & & & $100 \%$ & $64.25 \%$ \\
AtSAMS & & & & $100 \%$ \\
\hline
\end{tabular}

\section{Southem blot and expression analysis of PtSAMS at different phases of the life cycle}

To determine the copy number of SAMS in the P. tenera genome, genomic DNA of $P$. tenera was digested with restriction endonucleases Bam $\mathrm{HI}$ and $K p n \mathrm{I}$; then, Southern blot analysis was performed. After transferring to a membrane, the separated genomic DNA was hybridized to DIG-labeled PtSAMS gene-specific probe. Southern blot analysis result clearly showed that the PtSAMS gene was present as a single copy in P. tenera (Fig. 2). To examine the expression levels of PtSAMS transcripts at different life cycles, qPCR analysis was performed (Fig. 3). Total RNA was isolated from the conchocelis and thallus of $P$. tenera. The relative mRNA expression level in the thallus was higher than that in conchocelis by approximately three folds.

\section{Expression analysis of recombinant PtSAMS protein in $E$. coli by SDS-PAGE}

The complete ORF of the PtSAMS gene was cloned into a pET28(b) vector and expressed in the E. coli strain

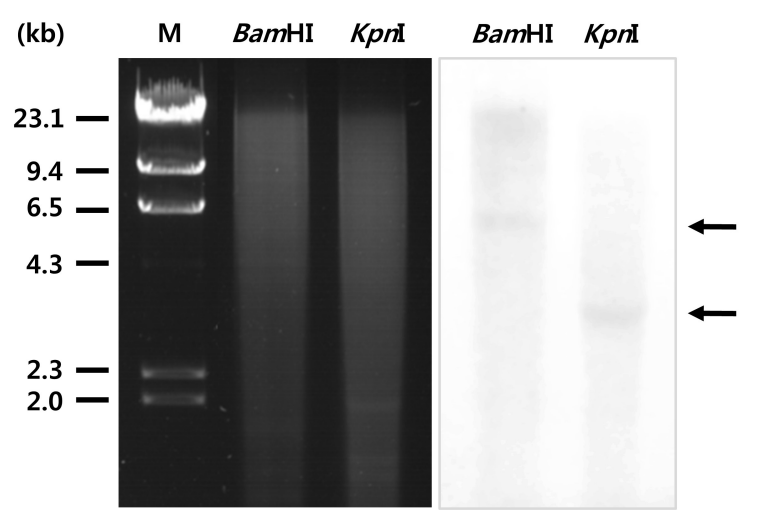

Fig. 2. Southern blot analysis of $P$. tenera genomic DNA: $5 \mu \mathrm{g}$ of genomic DNA per lane was digested with restriction enzymes and separated by electrophoresis in a $0.7 \%$ agarose gel. The DNA was transferred to a nylon membrane and probed with a DIG-labeled PtSAMS cDNA fragment. Molecular size markers are indicated on the left.

BL21(DE3). The empty pET28(b) vector was used as a control in this experiment. Expression of the recombinant PtSAMS protein was induced by adding $0.1 \mathrm{mM}$ IPTG after 1 hour; this reached a maximum at 7 hours (Fig. 4a). 
To confirm the production of His tag-fused PtSAMS, western blot analysis was carried out using anti-His (Fig. $4 b)$. The theoretical molecular weight of the recombinant PtSAMS was about $44.0 \mathrm{kDa}$.

\section{Growth of PtSAMS-expressing $E$. coli under abiotic stress}

To examine the effect of the overexpression of the PtSAMS protein in E. coli under various abiotic stresses, the recombinant cells were spotted on LB medium supplemented with $\mathrm{NaCl}$ and $\mathrm{KCl}$ (Fig. 5). The pET28(b)-

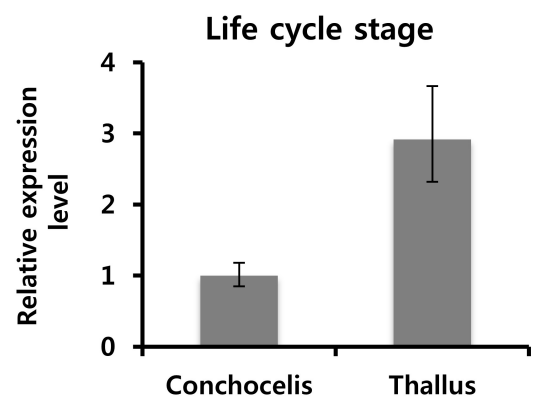

Fig. 3. Expression pattern analysis of PtSAMS at different life cycle stages in $P$. tenera: Total RNA was isolated from conchocelis and thallus of $P$. tenera at various life stages. The mRNA expression level of the conchocelis stage was set to 1. PtGAPDH was used as normalization control. The results are presented as average values with SD using each three times.

a

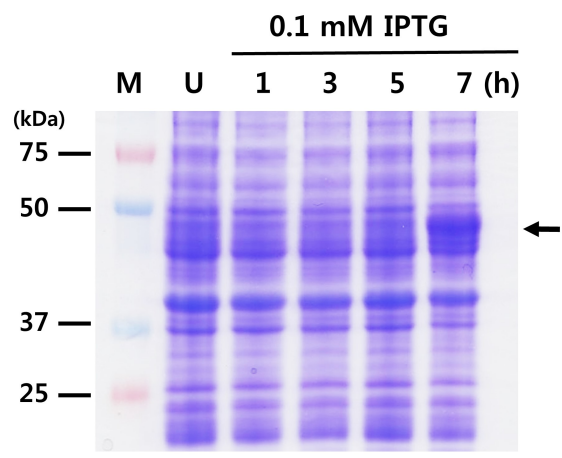

PtSAMS and pET28(b) cells showed similar growth on LB medium in overnight grown culture (Fig. 5a). The pET28(b)-PtSAMS recombinant cells showed increased number of colonies in $\mathrm{NaCl}$ and $\mathrm{KCl}$ treatment compared with that in control cells (Fig. 5b and 5c). In addition, the growth rate of recombinant cells was analyzed in LB liquid medium. pET28(b)-PtSAMS recombinant and pET28(b) empty vector control BL21(DE3) cells were inoculated into fresh LB liquid medium and medium supplemented with $\mathrm{NaCl}$ and $\mathrm{KCl}$. In LB liquid medium, pET28(b)PtSAMS recombinant cell and cells harboring the pET28(b)
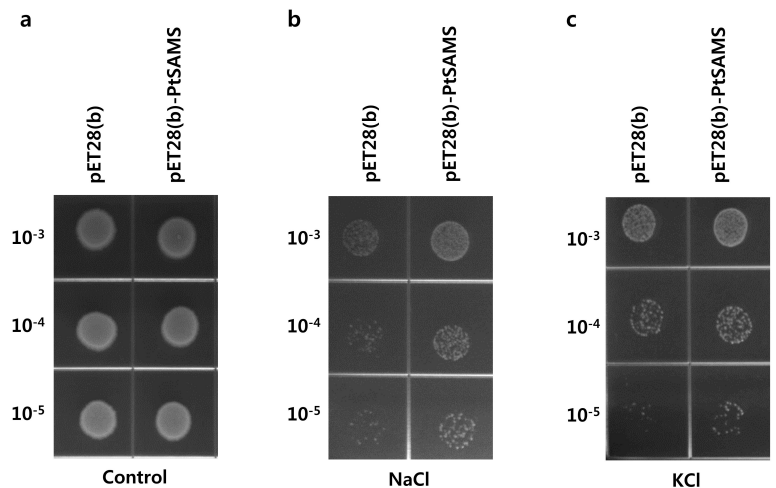

Fig. 5. Spot assay of pET28(b) and pET28(b)-PtSAMS recombinant protein in $E$. coli cells with $\mathrm{NaCl}$ and $\mathrm{KCl}$ treatment; $10 \mu \mathrm{L}$ from $10^{-3}$ to $10^{-5}$ dilution series were spotted on (a) control (LB-only) plate, or (b) LB plate supplemented with $400 \mathrm{mM}$ $\mathrm{NaCl}$, and (c) $400 \mathrm{mM} \mathrm{KCl}$.

b

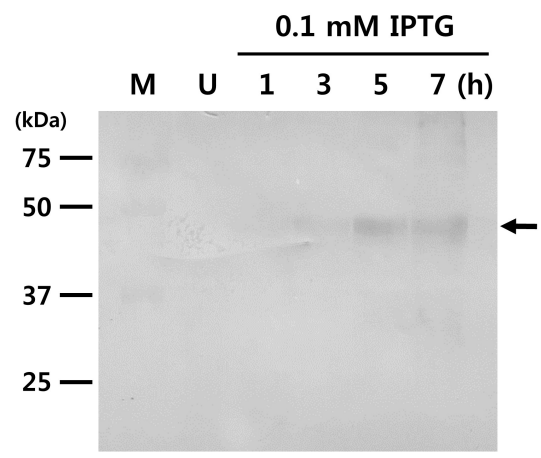

Fig. 4. SDS-PAGE and western blot analysis of PtSAMS recombinant protein in E. coli BL21(DE3). (a) SDS-PAGE analysis. M: marker, Lane 1: uninduced protein, Lane 2-4: induced protein by $0.1 \mathrm{mM}$ IPTG for 1, 3, 5 (hour), respectively. (b) Analysis of expression of recombinant PtSAMS protein in E. coli BL21(DE3) cells; western blotting analysis of the PtSAMS recombinant proteins with anti-His tag antibodies. Arrows indicate the His-tagged PtSAMS recombinant protein. 
vector alone showed similar growth at various times. In the $\mathrm{NaCl}$ - and KCl-treatment treatment, pET28(b)-PtSAMS recombinant cells showed better growth compared with cells harboring the vector alone 6 hours after inoculation (Fig. 6). Therefore, the spot and liquid culture assay indicated a similar pattern between the groups in terms of the abiotic stress response.

a

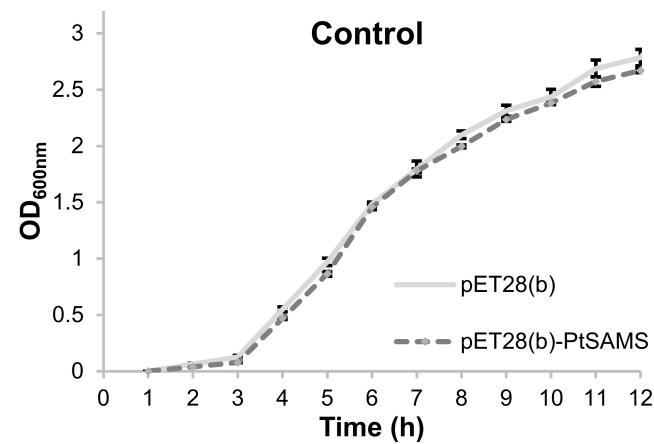

b

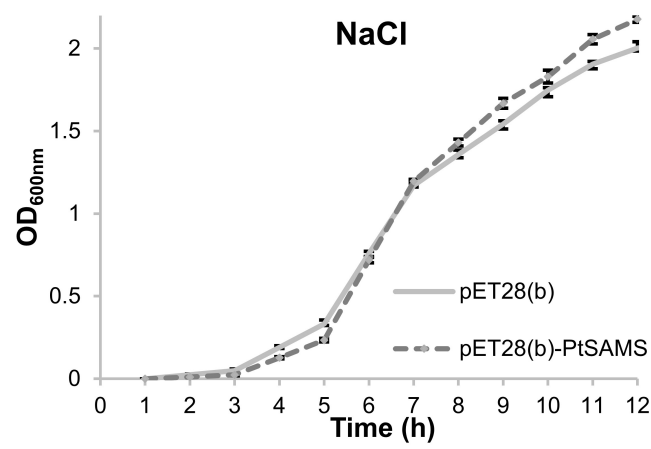

C

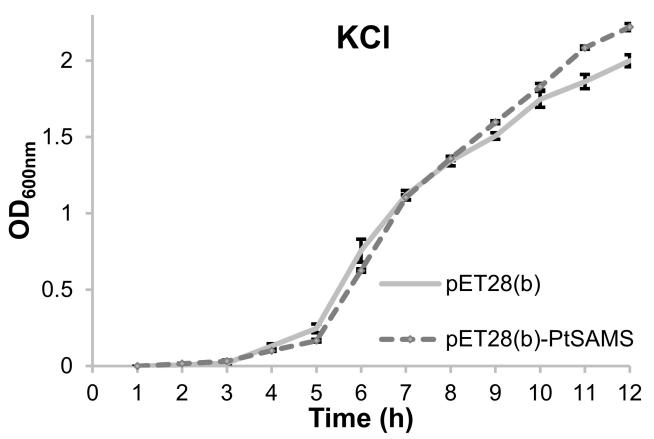

Fig. 6. Growth analysis of pET28(b) and pET28(b)PtSAMS recombinant protein in E. coli cells on liquid medium with $\mathrm{NaCl}$ and $\mathrm{KCl}$ treatment; (a) LB medium, (b) $400 \mathrm{mM} \mathrm{NaCl}$, and (c) $400 \mathrm{mM}$ $\mathrm{KCl} . \mathrm{OD}_{600}$ was recored at 1 hour interval up to 12 hours and mean values are represented in graph.

\section{DISCUSSION}

Pyropia grows in high intertidal zones where this plant experiences environmental conditions such as desiccation, salinity fluctuations, intense radiation, and high temperatures; additionally, Pyropia is regularly submerged in seawater and exposed to air during high and low tides (Xu et al. 2017). This plant has developed mechanisms to resist salt stress and survive under severe conditions. However, the molecular mechanisms underlying the salt stress tolerance in Rhodophyta are not fully understood. In this study, we attempted to isolate the salt stress gene in seaweed by cloning the $S$-Adenosyl-l-methionine synthetase (SAMS) gene from P. tenera; this gene was referred to as PtSAMS. S-Adenosyl-L-methionine synthase synthesizes S-adenosyl-L-methionine (AdoMet) from L-methionine and ATP in both prokaryotes and eukaryotes (Boerjan et al. 1994). In silico sequence alignment and phylogenetic analysis of amino acid sequence showed that PtSAMS comprises three conserved domains: the N-terminal domain, central domain, and C-terminal domain (Fig. 1a). Furthermore, PtSAMS contains two motifs, Met-binding and ATP-binding sites, that are highly conserved in other plants. In the phylogenetic tree based on amino acid sequences of different SAMS proteins, PtSAMS was clustered with the SAMS from G. sulphuraria, Rhodophyta (Fig. 1b). Taken together, these results suggest that PtSAMS from $P$. tenera is a member of the red algae SAMS family. Further, we identified the presence of a single copy of the SAMS gene in the Pyropia genome by DNA gel blot analysis (Fig. 2). To examine PtSAMS expression at various stages of the life cycle, RT-qPCR amplification of DNA from the conchocelis and thallus of $P$. tenera was performed (Fig. 3). The relative mRNA expression level of PtSAMS in the thallus was higher than in the conchocelis. However, the expression level did not differ under exposure to abiotic stresses in the thallus (data not shown). As Pyropia inhabits sea water, this organism is exposed to environmental stresses such as high salt levels and drought, and is therefore adapted for growth and survival in the marine environment (Xu et al. 2017). It is therefore possible that the expression of the PtSAMS gene is maintained at the level of a housekeeping gene owing to 
the constant exposure to such growing conditions.

Salinity stress is an important environmental factor that limits plant growth and development. SAM acts as a universal methyl group donor in biological processes during numerous specific trimethylations of protein, lipids, polysaccharides, and nucleic acids (Li et al. 2011). SAM additionally serves as precursor for polyamine biosynthesis (Evans and Malmberg 1989). The plant hormone, ethylene, is synthesized from SAM and participates in various physiological processes such as the stress response (Wang et al. 2002; Wang et al. 2013). SAMS is expressed in various tissues in other plant species (Li et al. 2011): in rice, the SAMS gene regulates ethylene-mediated inhibition of root development and alteration of cell wall structures (Fukuda et al. 2007). SAMS expression is associated with the mechanism underlying tolerance of plants to abiotic stresses; it has been shown that transgenic SAMS-expressing plants show enhanced resistance to abiotic stresses. In tomato, SAMS were differentially expressed after application with salt stress (Espartero et al. 1994). Overexpression of SAMS in M. sativa resulted in enhanced tolerance to cold stress by accelerating polyamine oxidation (Guo et al. 2014). In addition, the levels of three SAMS transcripts were increased transiently following the application of various stresses in Catharanthus roseus; in particular, a high degree of accumulation of SAM2 transcripts was observed (Schroder et al. 1997). Taken together with the results of previous studies, the present findings suggest that the expression of the SAMS gene maybe induced by a combination of ion toxicity and osmotic stress during exposure to salt stress.

E. coli growth assay results showed that pET8(b)PtSAMS recombinant E. coli cells showed better tolerance to salt stress than cells harboring the control vector (Fig. 5). Similar to our results, previous studies have reported that the expression of stress-induced genes enhances tolerance to various stresses in other plants. Overexpression of Lycoris radiata SAMS recombinant protein was induced effectively under high salinity $(\mathrm{NaCl}$ and $\mathrm{KCl})$ compared with that in control samples (Li et al. 2013; Cui et al. 2005). Ma et al. (2003) found that the expression of the SAMS gene from Suaeda salsa is induced under $\mathrm{NaCl}$ stress (Ma et al. 2003). Recently, it has been reported that the AvSAMS gene confers aluminum stress tolerance and facilitates epigenetic gene regulation (Ezaki et al. 2016). SAMS is therefore known to play roles in various plant defense systems. The present results indicate that PtSAMS maybe plays an important role in salt tolerance. This gene therefore suggests a potential bioresource for the genetic engineering of abiotic stress tolerance in plants.

\section{ACKNOWLEDGEMENTS}

This work was supported by a grant from the National Marine Biodiversity Institute Research Program (2017M 01300).

\section{REFERENCES}

Amme S, Matros A, Schlesier B, Mock HP. 2006. Proteome analysis of cold stress response in Arabidopsis thaliana using DIGE-technology. J. Exp. Bot. 57: 1537-1546.

Blouin NA, Brodie JA, Grossman AC, Xu P, Brawley SH. 2011. Porphyra: a marine crop shaped by stress. Trends Plant Sci. 16: 29-37.

Boerjan W, Bauw G, Van Montagu M, Inze D. 1994. Distinct phenotypes generated by overexpression and suppression of S-adenosyl-L-methionine synthetase reveal developmental patterns of gene silencing in tobacco. Plant Cell 6: 1401-1414.

Cui S, Huang F, Wang J, Ma X, Cheng Y, Liu J. 2005. A proteomic analysis of cold stress responses in rice seedlings. Proteomics 5: 3162-3172.

Espartero J, Pintor-Toro JA, Pardo JM. 1994. Differential accumulation of S-adenosylmethionine synthetase transcripts in response to salt stress. Plant Mol. Biol. 25: 217-227.

Evans JM, Malmberg RL. 1989. Do polyamines have roles in plant develoiment? Annu. Rev. Plant Physiol. Plant Mol. Biol. 40: 235-269.

Ezaki B, Higashi A, Nanba N, Nishiuchi T. 2016. An $\mathrm{S}$-adenosyl methionine synthetase (SAMS) gene from Andropogon virginicus L. confers aluminum stress tolerance and facilitates epigenetic gene regulation in Arabidopsis thaliana. Front. Plant Sci. 7: 1627. 
Fukuda T, Saito A, Wasaki J, Shinano T, Osaki M. 2007. Metabolic alterations proposed by proteome in rice roots grown under low $\mathrm{P}$ and high $\mathrm{Al}$ concentration under low pH. Plant Sci. 172: 1157-1165.

Gómez-Gómez L, Carrasco P. 1998. Differential expression of the S-adenosyl-L-methionine synthase genes during pea development. Plant Physiol. 117: 397-405.

Guo Z, Tan J, Zhuo C, Wang C, Xiang B, Wang Z. 2014. Abscisic acid, $\mathrm{H}_{2} \mathrm{O}_{2}$ and nitric oxide interactions mediated cold-induced S-adenosylmethionine synthetase in Medicago sativa subsp. falcata that confers cold tolerance through up-regulating polyamine oxidation. Plant Biotechnol. J. 12: 601-612.

Hua Y, Zhang BX, Cai H, Li Y, Bai X, Ji W, Wang ZY, Zhu YM. 2012. Stress-inducible expression of GsSAMS2 enhances salt tolerance in transgenic Medicago sativa. African Journal of Biotechnology 11: 4030-4038.

Kakinuma M, Nakamoto C, Kishi K, Coury DA, Amano H. 2017. Isolation and functional characterization of an ammonium transporter gene, PyAMT1, related to nitrogen assimilation in the marine macroalga Pyropia yezoensis (Rhodophyta). Mar. Environ. Res. 128: 76-87.

Kumar A, Taylor M, Altabella T, Tiburcio AF. 1997. Recent advances in polyamine research. Trends Plant Sci. 2: 124-130.

Lee MK, Kim IH, Choi YH, Choi JW, Kim YM, Nam TJ. 2015. The proliferative effects of Pyropia yezoensis peptide on IEC-6 cells are mediated through the epidermal growth factor receptor signaling pathway. Int. J. Mol. Med. 35: 909-914.

Li W, Han Y, Tao F, Chong K. 2011. Knockdown of SAMS genes encoding S-adenosyl-1-methionine synthetases causes methylation alterations of DNAs and histones and leads to late flowering in rice. J. Plant Physiol. 168: $1837-1843$

Li XD, Xia B, Wang R, Xu S, Jiang YM, Yu FB, Peng F. 2013. Molecular cloning and characterization of S-adenosylmethionine synthetase gene from Lycoris radiata. Mol. Biol. Rep. 40: 1255-1263.

Ma X, Wang Z, Qi Y, Zhao Y, Zhang H. 2003. Isolation of s-adenosylmethionine synthetase gene from Suaeda salsa and its differential expression under $\mathrm{NaCl}$ stress. Acta Botanica Sinica 45: 1359-1365.

Pajares MA, Markham GD. 2011. Methionine adenosyltransferase (s-adenosylmethionine synthetase). Adv.
Enzymol. Relat. Areas Mol. Biol. 78: 449-521.

Pulla RK, Kim YJ, Parvin S, Shim JS, Lee JH, Kim YJ, In JG, Senthil KS, Yang DC. 2009. Isolation of S-adenosyl-Lmethionine synthetase gene from Panax ginseng C.A. meyer and analysis of its response to abiotic stresses. Physiol. Mol. Biol. Plants 15: 267-275.

Qi YC, Wang FF, Zhang H, Liu WQ. 2010. Overexpression of suadea salsa S-adenosylmethionine synthetase gene promotes salt tolerance in transgenic tobacco. Acta Physiologiae Plantarum 32: 263-269.

Roeder S, Dreschler K, Wirtz M, Cristescu SM, van Harren FJ, Hell R, Piechulla B. 2009. SAM levels, gene expression of SAM synthetase, methionine synthase and ACC oxidase, and ethylene emission from $N$. suaveolens flowers. Plant Mol. Biol. 70: 535-546.

Schroder G, Eichel J, Breinig S, Schroder J. 1997. Three differentially expressed S-adenosylmethionine synthetases from Catharanthus roseus: molecular and functional characterization. Plant Mol. Biol. 33: 211-222.

Tester M, Bacic A. 2005. Abiotic stress tolerance in grasses. From model plants to crop plants. Plant Physiol. 137: 791-793.

Thompson JD, Higgins DG, Gibson TJ. 1994. CLUSTAL W: improving the sensitivity of progressive multiple sequence alignment through sequence weighting, positionspecific gap penalties and weight matrix choice. Nucleic Acids Res. 22: 4673-4680.

Uvackova L, Takac T, Boehm N, Obert B, Samaj J. 2012. Proteomic and biochemical analysis of maize anthers after cold pretreatment and induction of androgenesis reveals an important role of anti-oxidative enzymes. J. Proteomics 75: 1886-1894.

Wang F, Cui X, Sun Y, Dong CH. 2013. Ethylene signaling and regulation in plant growth and stress responses. Plant Cell Rep. 32: 1099-1109.

Wang KL, Li H, Ecker JR. 2002. Ethylene biosynthesis and signaling networks. Plant Cell 14 Suppl: S131-151.

Xu K, Xu Y, Ji D, Chen T, Chen C, Xie C. 2017. Cells tile a flat plane by controlling geometries during morphogenesis of Pyropia thalli. PeerJ 5: e3314.

Yadav NS, Rashmi D, Singh D, Agarwal PK, Jha B. 2012. A novel salt-inducible gene SbSI-1 from Salicornia brachiata confers salt and desiccation tolerance in $E$. coli. Mol. Biol. Rep. 39: 1943-1948.

Yang PF, Li XJ, Liang Y, Jing YX, Shen SH, Kuang TY. 
2006. Proteomic analysis of the response of Liangyoupeijiu (super high-yield hybrid rice) seedlings to cold stress. J. Integr. Plant Biol. 48: 945-951.
Yi LF, Wang P, Zhou X, Liu C. 2009. cDNA cloning and bioinformatic analysis of SAMS gene from Porphyra yezoensis. China Biotechnology 29: 43-49. 